Meta

Journal des traducteurs

Translators' Journal

\title{
Terminologie multilingue de l'énergie, évolution des techniques et défense de la langue française
}

\section{Kleinpeter}

Volume 34, numéro 3, septembre 1989

1. Actes du Colloque Les terminologies spécialisées : Approches quantitative et logico-sémantique et 2 . Actes du Colloque Terminologie et Industries de la langue

URI : https://id.erudit.org/iderudit/003324ar

DOI : https://doi.org/10.7202/003324ar

Aller au sommaire du numéro

Éditeur(s)

Les Presses de l'Université de Montréal

ISSN

0026-0452 (imprimé)

1492-1421 (numérique)

Découvrir la revue

Citer cet article

Kleinpeter, M. (1989). Terminologie multilingue de l'énergie, évolution des techniques et défense de la langue française. Meta, 34(3), 542-543.

https://doi.org/10.7202/003324ar d'utilisation que vous pouvez consulter en ligne. 


\section{TERMINOLOGIE MULTILINGUE DE L'ÉNERGIE, ÉVOLUTION DES TECHNIQUES ET DÉFENSE DE LA LANGUE FRANÇAISE}

M. KLEINPETER

Electricité de France

Paris, France

Le comité spécialisé «Terminologie de l'Énergie» de la Conférence Mondiale de l'Énergie, organisme qui regroupe près de 80 pays, a publié, à l'occasion du congrès de Cannes en 1986, la deuxième édition d'un glossaire multilingue couvrant l'ensemble des aspects du secteur de l'énergie.

Les deux langues officielles de la Conférence Mondiale de l'Énergie sont le français et l'anglais. L'ouvrage, cependant, a été édité en quatre langues: anglais, français, allemand et espagnol. Il est traduit dans un certain nombre d'autres versions linguistiques: portugais, arabe, hébreux, chinois, japonais, etc.

Le secteur énergétique ne constitue pas un ensemble isolé de l'économie, ainsi dans une section «généralisée», de nombreux concepts débordent le cadre strictement «physique» de l'énergie, et outre les aspects économiques tarifaires, des concepts relatifs à la formation des prix, du calcul des investissements, figurent au glossaire.

Une terminologie doit, sans être trop ambitieuse, pousuivre un but de standardisation des termes traités, pour qu'une notion soit comprise d'une manière «à peu près identique» dans toutes les langues. Ceci est parfois difficile, mais l'objectif doit en être recherché. Une bonne terminologie permet par exemple, l'expérience le prouve, d'harmoniser progressivement, pays par pays, les bilans et la comptabilité énergétiques. Pour l'économie mondiale, une telle approche devient pensable. Ainsi la section spécialisée «bilan et comptabilité énergétiques» a été publiée en version séparée et envoyée, grâce au soutien de l'UNESCO à de nombreux pays en voie de développement.

La qualification de certaines formes d'énergie, en «énergie non conventionnelle», «énergie nouvelle», «énergie renouvelable» et «énergie douce», peut conduire à des appréciations erronnées, des difficultés de compréhension, des abus de langage. Une politique énergétique dépasse nos frontières, requiert de tous les acteurs, une appréciation exacte des termes employés, de leur contenu, de leur impact, et même peut-on dire de leur résonnance médiatique.

L'énergie ne se limite pas uniquement à une mise en œuvre physique et économique. Le produit représente, sous une acuité plus ou moins forte, une agression pour l'environnement.

Le Comité Terminologie de la Conférence Mondiale de l'Énergie a cherché dans les quatre langues de base utilisées, à harmoniser le plus largement possible tous ces mêmes concepts, en mettant le lecteur en garde contre l'utilisation de termes équivoques. Nous en avons cité quelques-uns: énergie nouvelle, énergie renouvelable ou énergie douce.

Si les dénominations génériques sont sujettes à caution, les définitions relatives aux processus et aux équipements mêmes, ne le sont pas moins dans les domaines où le voca- 
bulaire n'a pas encore acquis la stabilité conférée par un long usage. C'est pouquoi, lorsque cela semble utile, des notes précisent les conditions d'application des concepts proposés.

L'évolution rapide de la terminologie nécessite des laps de temps de plus en plus courts entre les mises à jour et les compléments. Le Comité a convenu qu'après la publication de l'édition, une révision sera effectuée dans un horizon de six ans, après avoir recueilli l'ensemble des critiques et commentaires. Un nouveau dictionnaire multilingue de l'énergie sera préparé pour le Congrès qui aura lieu en Espagne en 1992. Cette édition commémorative du $500^{\mathrm{e}}$ anniversaire de la découverte de l'Amérique et de la mise en place du marché unique européen, est en voie de préparation. Elle sera publiée sous forme de livre sur support papier et sur disquettes. Grâce à un programme particulier, le Comité, avec l'aide de spécialistes de l'électronique et des logiciels spéciaux, espère pouvoir diffuser ces disquettes avec toutes les versions linguistiques dans lesquelles sera traduit le dictionnaire, y compris des versions de langues à idéogrammes. L'assistance de collègues d'Extrème-Orient a été confirmée au Comité pour atteindre cet objectif.

Un certain nombre d'experts du Comité sont d'origine française ou francophone (Canada, Belgique, Suisse et Afrique). Certains pays avaient demandé que le dictionnaire soit simplement présenté en langue anglaise et chacun le traduirait «comme cela lui convient» ou «à sa guise». Or, il s'est avéré, à travers une expérience de près de dix années, que pour le traitement d'un sujet sensible, une liste de concepts définis et un index alphabétique «en une seule langue», ne permettent pas de découvrir puis de cerner toutes les nuances. La préparation du travail en langue anglaise et française, et partiellement en allemand et en espagnol, a permis de saisir certaines différences. La confrontation de langues anglo-saxones avec les langues d'origine latine, enrichit au-delà de ce que l'on peut imaginer, un glossaire dont l'utilisation est faite au niveau planétaire. Il n'est pas dans les objectifs du Comité de défendre simplement un idiome, mais de démontrer que la langue française, avec les possibilités de contributions scientifiques, techniques des experts, permet une complémentarité dans l'élaboration du document. Celui-ci n'est pas uniquement destiné aux interprètes ou aux traducteurs, mais constitue, l'expérience nous le montre, une sorte de guide pratique de l'énergie facilitant la compréhension au niveau international.

Il permettra une assistance remarquée pour l'élaboration de certains actes commerciaux. Un dictionnaire technique en dix langues avec comme langues de base l'anglais et le français, ouvre des horizons immenses et permet d'éviter certains conflits pour faciliter la rédaction, par exemple, des contrats.

La langue française, dans ce contexte est sollicitée pour le niveau de sa précision, de sa rigueur face à d'autres langues.

Loin de tout sentiment empreint d'un chauvinisme par la volonté de voir présent son propre idiome, c'est la nécessité et l'utilité fondamentale qui ont fait pousuivre le Comité spécialisé de la Conférence Mondiale de l'Énergie. Est-il déplacé de dire que «nous défendons d'autant mieux dans cet organisme la langue française, que nous respectons, voire nous utilisons également les autres idiomes». Faut-il ajouter que très souvent en discutant dans une autre langue, la difficulté d'appréciation d'une nuance apparaît. Très souvent, nous nous retournons vers la version française pour essayer de trouver une solution acceptable par l'ensemble des membres du Comité.

Pour terminer, il faut mentionner que dix-sept pays sur les 80 pays membres de la Conférence Mondiale de l'Énergie et de grands organismes internationaux, comme l'UNESCO, l'OCDE, la CEI, l'OPEP, et l'UNIPEDE, participent aux travaux et contribuent au succès de l'entreprise. 